\title{
Can Innovative Learning Affect Students' HOTS Achievements?: A Meta-Analysis Study
}

\author{
Duden Saepuzaman ${ }^{1 *}$, Heri Retnawati ${ }^{2}$, Edi Istiyono ${ }^{3}$, Haryanto ${ }^{4}$ \\ ${ }^{1}$ Graduate School, Educational Research and Evaluation program, Universitas Negeri Yogyakarta, Jl. Colombo, Yogyakarta, Indonesia \\ (as student doctoral program); Physisc Education Department, Universitas Pendidikan Indonesia, J. Dr. Setiabudhi, Bandung, \\ Indonesia (as Lecturer) \\ ${ }^{2-4}$ Universitas Negeri Yogyakarta, Jl. Colombo No. 1, Yogyakarta 55281, Indonesia
}

\begin{abstract}
This study looks at innovative learnings' effect on achieving students' High Order Thinking Skills (HOTS). HOTS is one part of the skills that need to be developed in the 21 st Century. This research is a quantitative study with a meta-analysis approach to the mean with random-effects models. Generally, the steps involve formulating research problems meta-analysis to be performed, collecting studies, calculating the effect size, test of heterogeneity, data analysis (summary effect, forest plots, funnel plots, test the potential for publication bias), and concludes. The research sample of 42 research includes international journals and national, international, and nationwide proceedings and a thesis student. The analysis using random models (random-effect model) obtained a mean value of the effect size of aggregation or summary effect amounted to 77.37, standard error weighs the mean 12.36, the upper limit of 53.14, and the lower limit of 101.60. Analysis of the null hypothesis (Ho: actual effect $\boldsymbol{\theta}=$ 0) leads to rejecting Ho because the $Z$ value of the summary effect is 77.37 with the one-tailed p-value (0.00) smaller than the a value (0.05). Analysis of publication bias tests from Funnel Plot, Rank Correlation, Regression Method outputs, and "Trim and Fill" shows no potential publication bias regarding conclusions drawn. So the findings made based on the random effect model on the application of innovative learning affect HOTS students achievement (analyzing, evaluating, creating, critical and creative thinking skill aspects) are valid. The implication is that teachers or other practitioners can use innovative learning to improve students' HOTS.
\end{abstract}

Keywords: Innovative learning, HOTS, meta-analysis

\section{INTRODUCTION}

Today it is known as a century full of competent challenges both in science and technology. The competency needed in the 21st Century focuses on conceptual knowledge and the skills to apply knowledge and thinking skills. The 21st Century skills one has several definitions (Aljarrah \& Khataybeh, 2021). The 21st Century skills is a group of skills that include several skills, including life skills, workforce skills, interpersonal skills, practical skills, and non-cognitive skills (Silva, 2009). On the other hand, the 21st Century skills were defined as the skills that the learner needs to succeed in their professional life through the information age. It was divided into three categories: First: Learning skills and called (4C): Critical thinking, Creativity, Collaboration, and Communication (Stauffer, 2018). Critical and creative thinking are high-level skills (High Order Thinking Skills, HOTS) (Conklin, 2012; King et al., 2010; Krulik \& Rudnick, 1999; Presseisen, 1988).

Some experts associate HOTS with a type of thinking skill that each individual can perform. Thinking skills part of HOTS, according to experts, include critical thinking skills and creative thinking (Conklin, 2012; King et al., 2010; Krulik \& Rudnick, 1999; Presseisen, 1988), problemsolving (Brookhart, 2010; Presseisen, 1988), logical thinking, reflective, and metacognitive (King et al., 2010), and decision making(Presseisen, 1988). These skills are not foreign terms in the learning process; they have become targets and are part of each subject's learning objectives (Jailani \& Retnawati, 2016) which used pretest-posttest experimental non-equivalent control group. Experimental class was a class which was taught by using problem-based learning, while the control class was a class which was taught by using direct instruction. The population of this research was the seventh graders of several Junior High Schools in DI Yogyakarta which have implemented problem based learning. The samples of this research were 515 students of Junior High School students from 10 schools in four districts and one city in Yogyakarta.

Corresponding Author e-mail: dudensaepuzaman.2019@student. uny.ac.id

https://orcid.org/0000-0002-7810-2328

How to cite this article: Saepuzaman $D$, Retnawati $H$, Istiyono $E$, eHaryanto, (2021). Can Innovative Learning Affect Students' HOTS Achievements?: A Meta-Analysis Studys. Pegem Journal of Education and Instruction, Vol. 11, No. 4, 2021, 290-305

Source of support: Nil

Conflict of interest: None.

DOI: $10.47750 /$ pegegog.11.04.28

Received: 26.07.2021

Accepted: 23.09.2021 Publication: 01.10.2021 
The schools from which the samples were taken were both public and private schools which were selected based on their achievement in the national examination. The schools were selected using stratified random sampling, while the classes were selected randomly. The quantitative data analysis was conducted by using both descriptive and inferential statistic. The results showed that: $(1$.

In the context of learning in school, the HOTS indicator refers to Bloom's taxonomy put forward by Benjamin S. Bloom in 1956 (Crumb, 1983). When associated with cognitive processes in Bloom's taxonomy, HOTS contrasted with LOTS (Lower Order Thinking Skills). Cognitive process analysis (analysis), synthesis (synthesis), and evaluation ( evaluation) were categorized as HOTS, while knowledge (knowledge), understanding (comprehension), and applications (application ) were included as LOTS (Fisher, 2010). Still related to HOTS and LOTS' categorization in Bloom's taxonomy, a different opinion was expressed by Thompson (Thompson, 2011), who categorized the analysis, synthesis, and evaluation as HOTS, knowledge, and understanding as LOTS, while the application classified as HOTS or LOTS. Bloom's taxonomy revision by Anderson \& Krathwohl (Anderson \& Krathwohl, 2001), where learning objectives classification into two dimensions: cognitive processes and knowledge, HOTS in Bloom's taxonomy need to be adjusted. Based on Bloom's taxonomy revisions (Anderson \& Krathwohl, 2001), HOTS's dimension includes the process of analyzing (analyze ), evaluating (Evaluate), and create ( create ) (X. Liu, 2014), while the dimensions of knowledge HOTS include conceptual knowledge, procedural knowledge, and metacognitive knowledge.

HOTS is very important for everyone, including students as the next generation. The implication, the implementation of education or learning in the classroom must facilitate students' high-level thinking skills. Of course, achieving this goal requires cooperation, support, and effort from all parties involved, especially teachers. Teachers should create the right and training HOTS on students in both the learning and assessment.

Teachers must be able to plan and implement the learning that can facilitate HOTS students' achievement optimally. The learning carried out must focus on students no longer concentrate on the teacher as is usually the case in the field. Learning that is not, as usual, is done and aims to facilitate students in building their knowledge so that more optimal learning is usually known as the term innovative learning. The use of innovative words in learning is still general. In the context of the learning process can be many learning models that fall into the category of innovative learning, such as instructional learning, learning with a scientific approach (scientific approach), Problem Based Learning (PBL), Learning-Based Autonomy Learner, Scientific Approach, Problem Solving Strategy, contextual learning, inquiry learning, and other learning. In addition to a review of the learning process or model, innovative learning can be view in terms of the media used, such as interactive media, video simulations or animations, the use of electronic teaching materials, or others.

Many studies state that certain learning models can facilitate the improvement of students' HOTS achievement. Problem-based learning can facilitate the achievement of students' HOTS (Arends \& Kilcher, 2010; Guedri, 2001). The inquiry learning model has a positive effect on students higher order thinking skills (Hendryarto, 2013; Smart \& Marshall, 2013). These studies are very limited in their applicability only to certain samples or characteristics of students. Thorough research that compiles all learningrelated outcomes that are able to facilitate student HOTS is still limited.In this study, the focus is on studies related to innovative learning on HOTS student achievement. This study uses the meta-analysis approach, a part of quantitative research using secondary data from studies that already exist and have been used by other researchers who carried out systematically and quantitatively to get accurate conclusions (Retnawati, 2018). Thus, in this study, the initial stages are collecting material in the form of research -Research relevant to the achievement of HOTS innovative learning in students of existing research publications covering international journals and national, proceedings of international and national and theses dissertations of students. The next step is analysis in the meta-analysis until an accurate (valid) conclusion.

\section{Method \\ Research Design}

This research is a quantitative study using a meta-analysis approach to analyze empirical studies conducted by previous researchers regarding the effect of applying innovative learning in students' HOTS achievements, quantitative research results, research results in comparable form, for example, in this case, on a mean. The study results are used as material to calculate the effect size, which compiles the aggregate. The meta-analysis was used to test constructs and relationships compared. This meta-analysis is a particular research method for combining studies that can measure their effect size. Value effect size is used to achieve the standard value in evaluating independent studies' results with meta-analysis (Turgut \& Turgut, 2018). The effect size value also provides a standardized independent study result and is assessed based on the same criteria (Turgut \& Turgut, 2018). The studies that are collected henceforth were coded based on specific criteria. Encoding is the process of extracting data from the individual studies to obtain data that the air responds late with the data analyzed (Çoğaltay \& Karadağ, 2015; Koza Çiftçi \& Yıldız, 2019). 
Furthermore, meta-analysis research using statistical analysis in research and interpreting findings (Pigott, 2012). In this research, the meta-analysis used is a meta-analysis with the mean. So the determination of the effect size is analyzed using the mean and requires a mean value and standard deviation.

\section{Population and Sample/ Study Group/Participants}

The research sample of 42 research includes international journals and national, international, and nationwide proceedings and a thesis student whose work is related to implementing innovative teaching-learning in HOTS student achievement. Reference selection is based on the validity of the data and the availability of the required information. Of all the references used, the application of the learning model is generally able to facilitate the improvement of students' HOTS achievement with specific characteristics of students employed as research samples. This research is to see a comprehensive picture of the application of innovative learning models in facilitating students' HOTS.

\section{Data Collection Tools}

The selection of research samples so that 42 research results were taken related to the application of innovative learning in the achievement of students' HOTS were carried out by considering the validity data and the availability of the required information. The computer program JASP 0.11.1.0 was used (free from the page: https://jasp-stats.org/previousversions/). With the help of JASP, summary effects, forest plots and funnel plots will be obtained. The following analysis step is to interpret the output summary effect, forest plot and funnel plot. To detect the existence of publication bias in terms of Funnel Plot, Rank Correlation and Regression Method, and Trim and Fill.

\section{Data Collection}

The data in this study are secondary. This secondary data is in the form of studies or relevant research results as material for meta-analysis. Research data collection is done by searching for online international journals, a thesis, or a dissertation. The priority of the search focuses on research published in reputable international journals. But some studies do not include standard deviations as data needed in a meta-analysis. Other investigations are through university repositories or libraries that provide theses and dissertations as research students and supervisors. Even though the final project does not include the mean and standard deviation, it can be determined from the original score attached. From this search, 42 studies were relevant to the criteria.

From 42 research search results, the next step is to do the coding. This study's coding is researcher/study, year, independent variable (the type of innovative learning), dependent variable (HOTS), sample size, mean achievement, and standard deviation. This stage is part of the analysis before the statistical analysis process.

\section{Data Analysis}

\section{Determination of standard effect sizes (transformed)}

After obtaining mean data and standard deviations for each study, the next step is to transform the mean into the same scale, resulting in each study's effect size. In this study, the range of values from 1 to 100 , the transformation carried out refers to a scale of 1-100. This transformation is to standardize the effect size of each study. Standard effect sizes are used to compare independent group means, which are considered comparable for each study of each of the two variables (Freeman et al., 1986; Koza Çiftçi \& Yıldız, 2019)and (ii.

\section{Heterogeneity Test}

After calculating the effect size, what needs to calculate the aggregation effect size, which is also called the summary effect, is conducting a meta-analysis. In the aggregate calculation, there are two models to choose from, namely the fixed model (fixed-effect model) and models random (random effect model) (Retnawati, Apino, et al., 2018). To determined the models, the heterogeneity test is needed. Heterogeneity test using the

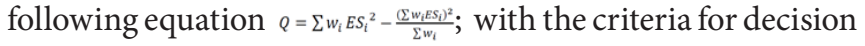
making statistically, Ho is rejected if the chi-square table. Ho here states that the effect size $(\overline{E S})$ between homogeneous studies (Retnawati, Apino, et al., 2018).

\section{Final Data analysis}

Data analysis included a summary effect, forest plot, funnel plot, and publication bias. Summary effect $(\overline{E S})$ can get manually or computer-assisted. Manually using equations $\overline{E S}=\frac{\sum w_{i} E S_{t}}{\sum w_{t}}$ (Retnawati, Apino, et al., 2018), while computer programs using the JASP 0.11.1.0 program ( free obtained from https://jasp-stats.org/previous-versions/). Aided by JASP will get a summary effect, forest plot, and funnel plot. The subsequent analysis is the interpretation output summary effect, forest plots, and funnel plots. To detect the publication bias in the Funnel plot, Rank Correlation and Regression Method, and Trim and Fill.

\section{FINDINGS}

Of the 42 studies deemed relevant to the search results, various characteristics started from the year, sample size, mean values, innovative learning models (independent variables ), and HOTS's definition (dependent variable). In general, research results are determined by researchers as samples presented in Table 1.

0Based on the table above, there are 42 relevant studies. If we look at the mean value, we will see a slightly different 
mean value, and this is because of the different scales used. The next step is to transform the mean into the same scale, and the result is an effect size of each study. Because the most common is a score range of 1 to 100 , other scores convert to a scale of 1-100. The standard deviation is also transformed to calculate $S E$ and $w$. The results are presented in Table 2.
In conducting a meta-analysis, after calculating the effect size, what needs to be done is to calculate the aggregation effect size, also called the summary effect. In the aggregate calculation, there are two models to choose from, namely the fixed model ( fixed-effect model ) and models random ( random effect model ) (Retnawati, Apino, et al., 2018). To determine which model fit, using the heterogeneity test-the

Table 1: Results of relevant research (study) findings

\begin{tabular}{|c|c|c|c|c|c|}
\hline Study & Research er & $N$ & Mean & $\begin{array}{l}\text { Independent Variable (Innovative } \\
\text { Learning) }\end{array}$ & $\begin{array}{l}\text { Dependent Variable(High } \\
\text { Order Thinking Skill, HOTS) }\end{array}$ \\
\hline Study_1 & (S. Liu et al., 2018) & 44 & 255.11 & Constructivism & $\begin{array}{l}\text { Problem Solving and Critical } \\
\text { Thinking }\end{array}$ \\
\hline Study_2 & (Salam \& Miriam, 2016) & 34 & 78.20 & Learner Autonomy based learning & $\begin{array}{l}\text { Analyzing (C4), evaluating } \\
\text { (C5), and creating (C6) }\end{array}$ \\
\hline Study_3 & (Folly Eldy \& Sulaiman, 2013) & 28 & 44.02 & Integrated PBL Approach & $\begin{array}{l}\text { Critical Thinking and } \\
\text { Creative-Critical Thinking }\end{array}$ \\
\hline Study_4 & (Khoiriah \& Jalmo, 2020) & 29 & 81.38 & $\begin{array}{l}\text { Student Worksheets Based On } \\
\text { Discovery Learning }\end{array}$ & $\begin{array}{l}\text { Analysis (C4), evaluate } \\
\text { (C5), and creating (C6) }\end{array}$ \\
\hline Study_5 & (Luthfiyah et al., 2019) & 30 & 77.18 & $\begin{array}{l}\text { Problem Based Learning (PBL) } \\
\text { learning model in curriculum } 2013 \\
\text { using the development of teaching } \\
\text { materials }\end{array}$ & analytical and creative \\
\hline Study_6 & (Utomo et al., 2019) & 32 & 76.94 & $\begin{array}{l}\text { Problem Based Learning with a } \\
\text { Scientific Approach }\end{array}$ & $\begin{array}{l}\text { Analyzing (C4), evaluating } \\
(\mathrm{C} 5) \text {, and creating (C6) }\end{array}$ \\
\hline Study_7 & (Saputri et al., 2019) & 32 & 82.61 & $\begin{array}{l}\text { Stimulating Higher Order Thinking } \\
\text { Skills Model }\end{array}$ & Critical Thinking \\
\hline Study_8 & (Malik et al., 2017) & 20 & 7.40 & HOT lab design & creative thinking skills \\
\hline Study_9 & (Ambarita et al., 2019) & 338 & 84.60 & $\begin{array}{l}\text { Group Investigation Based on Hands- } \\
\text { on Activities }\end{array}$ & $\begin{array}{l}\text { Analysis, evaluation, and } \\
\text { creation; Logical reasoning } \\
\text { Decision and critical } \\
\text { thinking; Problem-solving; } \\
\text { Creativity and creative } \\
\text { thinking }\end{array}$ \\
\hline Study_10 & Tambunan & 138 & 75.60 & $\begin{array}{l}\text { Problem Solving Strategy and the } \\
\text { Scientific Approach }\end{array}$ & $\begin{array}{l}\text { communication, creativity, } \\
\text { problem-solving and } \\
\text { mathematical reasoning }\end{array}$ \\
\hline Study_11 & (Hidayati \& Retnawati, 2018) & 30 & 51.33 & $\begin{array}{l}\text { Problem Based Learning and scientific } \\
\text { approach }\end{array}$ & $\begin{array}{l}\text { Analyzing (C4), evaluating } \\
\text { (C5), and creating (C6) }\end{array}$ \\
\hline Study_12 & (Saregar et al., 2016) & 26 & 68.30 & CUPS Learning & $\begin{array}{l}\text { analyzing (C4), evaluating } \\
(\mathrm{C} 5) \text {, and creating (C6) }\end{array}$ \\
\hline Study_13 & (Fauziah, 2013) & 31 & 87.62 & Online problem-based learning & $\begin{array}{l}\text { Creativity and Critical } \\
\text { Thinking }\end{array}$ \\
\hline Study_14 & (Ramadhan, 2019) & 34 & 83.61 & Physics Comic with Android & $\begin{array}{l}\text { analyzing }(\mathrm{C} 4) \text {, evaluating } \\
(\mathrm{C} 5) \text {, and creating }(\mathrm{C} 6)\end{array}$ \\
\hline Study_15 & (Dasilva \& Suparno, 2019) & 106 & 75.14 & $\begin{array}{l}\text { Interactive Physics Mobile Learning } \\
\text { Media (IPMLM) with Scaffolding } \\
\text { learning }\end{array}$ & $\begin{array}{l}\text { analyzing (C4), evaluating } \\
(\mathrm{C} 5) \text {, and creating (C6) }\end{array}$ \\
\hline Study_16 & (Sekarini, 2019) & 33 & 82.42 & $\begin{array}{l}\text { Contextual based Science Outdoor } \\
\text { Learning }\end{array}$ & $\begin{array}{l}\text { analyzing }(\mathrm{C} 4) \text {, evaluating } \\
\text { (C5), and creating (C6) }\end{array}$ \\
\hline
\end{tabular}




\begin{tabular}{|c|c|c|c|c|c|}
\hline Study & Researcher & $N$ & Mean & $\begin{array}{l}\text { Independent Variable (Innovative } \\
\text { Learning) }\end{array}$ & $\begin{array}{l}\text { Dependent Variable(High } \\
\text { Order Thinking Skill, HOTS) }\end{array}$ \\
\hline Study_17 & (Yusuf, 2019) & 19 & 68.26 & $\begin{array}{l}\text { Higher Order Thinking Skills Learning } \\
\text { Model }\end{array}$ & $\begin{array}{l}\text { Critical thinking, creative } \\
\text { thinking, and problem- } \\
\text { solving }\end{array}$ \\
\hline Study_18 & (Ferty \& Suparno, 2019) & 90 & 75.34 & $\begin{array}{l}\text { Android Based Interactive Physics } \\
\text { Mobile Learning Media }\end{array}$ & $\begin{array}{l}\text { analyzing }(\mathrm{C} 4) \text {, evaluating } \\
(\mathrm{C} 5) \text {, and creating }(\mathrm{C} 6)\end{array}$ \\
\hline Study_19 & (Haryanto \& Arty, 2019) & 30 & 15.53 & $\begin{array}{l}\text { Science video animation based } \\
\text { Contextual Teaching And Learning } \\
\text { (CTL) }\end{array}$ & $\begin{array}{l}\text { analyzing }(\mathrm{C} 4) \text {, evaluating } \\
(\mathrm{C} 5) \text {, and creating }(\mathrm{C} 6)\end{array}$ \\
\hline Study_20 & (Puspaningtyas, 2019) & 18 & 83.61 & Enhancing thinking skill strategy & $\begin{array}{l}\text { analyzing }(\mathrm{C} 4) \text {, evaluating } \\
(\mathrm{C} 5) \text {, and creating }(\mathrm{C} 6)\end{array}$ \\
\hline Study_21 & (Arisandi \& Sutrisno, 2019) & 32 & 70.66 & Analogy lerarning & $\begin{array}{l}\text { analyzing }(\mathrm{C} 4) \text {, evaluating } \\
(\mathrm{C} 5) \text {, and creating (C6) }\end{array}$ \\
\hline Study_22 & (Suleman \& Sugijarto, 2019) & 29 & 76.45 & $\begin{array}{l}\text { Three Dimension Visualization with } \\
\text { Virtual Reality (3D-VR) }\end{array}$ & Critical thinking skill \\
\hline Study_23 & (Ariansyah \& Soenarto, 2019) & 21 & 56.90 & $\begin{array}{l}\text { Contextual Teaching and Learning } \\
\text { (CTL) }\end{array}$ & Critical thinking skill \\
\hline Study_24 & (Alandia \& Suparwoto, 2019) & 61 & 92.10 & $\begin{array}{l}\text { Problem Based Learning With Web in } \\
\text { Physics Learning }\end{array}$ & Critical thinking skill \\
\hline Study_25 & (Kurnia \& Retnowati, 2019) & 25 & 3.52 & $\begin{array}{l}\text { Erroneous worked example and } \\
\text { grouping strategy }\end{array}$ & $\begin{array}{l}\text { analyzing }(\mathrm{C} 4) \text {, evaluating } \\
(\mathrm{C} 5) \text {, and creating }(\mathrm{C} 6)\end{array}$ \\
\hline Study_26 & (Baskoro, 2019) & 17 & 86.76 & $\begin{array}{l}\text { Variation Theory in mathematic } \\
\text { learning }\end{array}$ & $\begin{array}{l}\text { analyzing }(\mathrm{C} 4) \text {, evaluating } \\
(\mathrm{C} 5) \text {, and creating }(\mathrm{C} 6)\end{array}$ \\
\hline Study_27 & (Wardani, 2019) & 64 & 83.48 & Guided inquiry Learning & Critical thinking skill \\
\hline Study_28 & (Putri \& Ghufron, 2019) & 86 & 79.36 & The Power Of Two Strategy & Critical thinking skill \\
\hline Study_29 & (Maghfiroh \& Mulyani, 2019) & 35 & 86.43 & HOTS based Problem Based Learning & Critical thinking skill \\
\hline Study_30 & (Ferty \& Suparno, 2019) & 90 & 69.50 & $\begin{array}{l}\text { Android-based Interactive Physics } \\
\text { Mobile Learning Media (IPMLM) }\end{array}$ & $\begin{array}{l}\text { analyzing }(\mathrm{C} 4) \text {, evaluating } \\
(\mathrm{C} 5) \text {, and creating }(\mathrm{C} 6)\end{array}$ \\
\hline Study_31 & (Widiyowati, 2014) & 42 & 62.74 & Contextual Learning Model & Critical thinking skill \\
\hline Study_32 & (Rahayu \& Utaminingsih, 2017) & 50 & 4.87 & Effective Questioning strategy & $\begin{array}{l}\text { analyzing }(\mathrm{C} 4) \text {, evaluating } \\
\text { (C5), and creating (C6 }\end{array}$ \\
\hline Study_33 & (Redhana, 2013) & 70 & 24.93 & $\begin{array}{l}\text { Problem-based Learning and Socratic } \\
\text { question }\end{array}$ & Critical thinking skill \\
\hline Study_34 & (Suarsana, 2013) & 34 & 27.60 & Problem Solving based E-module & Critical thinking skill \\
\hline Study_35 & (Mayasari \& Adawiyah, 2016) & 24 & 78.20 & Problem Based Learning & $\begin{array}{l}\text { analyzing }(\mathrm{C} 4) \text {, evaluating } \\
(\mathrm{C} 5) \text {, and creating (C6) }\end{array}$ \\
\hline Study_36 & J (Hendryarto, 2013) & 28 & 85.71 & Inquiry-Based Learning & $\begin{array}{l}\text { analyzing }(\mathrm{C} 4) \text {, evaluating } \\
(\mathrm{C} 5) \text {, and creating }(\mathrm{C} 6)\end{array}$ \\
\hline Study_37 & (Erny et al., 2017) & 37 & 84.46 & Scientific Approach & Critical and creative thinking \\
\hline Study_38 & (Nyoman Setiawan, 2015) & 42 & 59.21 & $\begin{array}{l}\text { Science Contextual Learning integrated } \\
\text { with higher-order thinking skill }\end{array}$ & Critical and creative thinking \\
\hline Study_39 & (Rosida et al., 2017) & 30 & 62.85 & Interactive e-book based learning & Critical thinking skill \\
\hline Study_40 & (Najib, 2015) & 36 & 78.34 & $\begin{array}{l}\text { Phet Simulation in inquiry laboratory } \\
\text { learning }\end{array}$ & $\begin{array}{l}\text { analyzing }(\mathrm{C} 4) \text {, evaluating } \\
(\mathrm{C} 5) \text {, and creating (C6) }\end{array}$ \\
\hline Study_41 & (Azis Nur, 2016) & 14 & 62.50 & Problem Based Learning & $\begin{array}{l}\text { analyzing }(\mathrm{C} 4) \text {, evaluating } \\
(\mathrm{C} 5) \text {, and creating }(\mathrm{C} 6)\end{array}$ \\
\hline Study_42 & (Agustina et al., 2020) & 42 & 62.74 & Contextual teaching and learning & Critical thinking skill \\
\hline
\end{tabular}


Table 2: Tabulation of transformed data and data for data analysis

\begin{tabular}{|c|c|c|c|c|c|c|c|c|}
\hline Study & $n$ & mean & $s$ & $\min$ & $\max$ & ES (transf) & $s$ (transf) & $S E$ \\
\hline Study_1 & 44 & 255.11 & 19.16 & 0 & 300 & 85.04 & 6.39 & 0.96 \\
\hline Study_2 & 34 & 78.20 & 9.40 & 0 & 100 & 78.20 & 9.40 & 1.61 \\
\hline Study_3 & 28 & 44.02 & 11.40 & 0 & 60 & 73.37 & 19.00 & 3.59 \\
\hline Study_4 & 29 & 81.38 & 6.53 & 0 & 100 & 81.38 & 6.53 & 1.21 \\
\hline Study_5 & 30 & 77.18 & 9.37 & 0 & 100 & 77.18 & 9.37 & 1.71 \\
\hline Study_6 & 32 & 76.94 & 15.67 & 0 & 100 & 76.94 & 15.67 & 2.77 \\
\hline Study_7 & 32 & 82.61 & 8.07 & 0 & 100 & 82.61 & 8.07 & 1.43 \\
\hline Study_8 & 20 & 7.40 & 0.60 & 0 & 10 & 74.00 & 6.00 & 1.34 \\
\hline Study_9 & 338 & 84.60 & 6.58 & 0 & 100 & 84.60 & 6.58 & 0.36 \\
\hline Study_10 & 138 & 75.60 & 6.85 & 0 & 100 & 75.60 & 6.85 & 0.58 \\
\hline Study_11 & 30 & 51.33 & 7.97 & 0 & 60 & 85.55 & 13.28 & 2.43 \\
\hline Study_12 & 26 & 68.30 & 11.90 & 0 & 100 & 68.30 & 11.90 & 2.33 \\
\hline Study_13 & 31 & 87.62 & 20.28 & 0 & 100 & 87.62 & 20.28 & 3.64 \\
\hline Study_14 & 34 & 83.61 & 5.80 & 0 & 100 & 83.61 & 5.80 & 0.99 \\
\hline Study_15 & 106 & 75.14 & 8.03 & 0 & 100 & 75.14 & 8.03 & 0.78 \\
\hline Study_16 & 33 & 82.42 & 7.19 & 0 & 100 & 82.42 & 7.19 & 1.25 \\
\hline Study_17 & 19 & 68.26 & 4.62 & 0 & 100 & 68.26 & 4.62 & 1.06 \\
\hline Study_18 & 90 & 75.34 & 4.56 & 0 & 100 & 75.34 & 4.56 & 0.48 \\
\hline Study_19 & 30 & 15.53 & 1.69 & 0 & 20 & 77.65 & 8.45 & 1.54 \\
\hline Study_20 & 18 & 83.61 & 6.59 & 0 & 100 & 83.61 & 6.59 & 1.55 \\
\hline Study_21 & 32 & 70.66 & 14.10 & 0 & 100 & 70.66 & 14.10 & 2.49 \\
\hline Study_22 & 29 & 76.45 & 7.09 & 0 & 100 & 76.45 & 7.09 & 1.32 \\
\hline Study_23 & 21 & 56.90 & 5.90 & 0 & 64 & 88.91 & 9.22 & 2.01 \\
\hline Study_24 & 61 & 92.10 & 4.92 & 0 & 100 & 92.10 & 4.92 & 0.63 \\
\hline Study_25 & 25 & 3.52 & 0.78 & 0 & 5 & 70.40 & 15.60 & 3.12 \\
\hline Study_26 & 17 & 86.76 & 4.20 & 0 & 100 & 86.76 & 4.20 & 1.02 \\
\hline Study_27 & 64 & 83.48 & 13.42 & 0 & 100 & 83.48 & 13.42 & 1.68 \\
\hline Study_28 & 86 & 79.36 & 8.97 & 0 & 100 & 79.36 & 8.97 & 0.97 \\
\hline Study_29 & 35 & 86.43 & 5.74 & 0 & 100 & 86.43 & 5.74 & 0.97 \\
\hline Study_30 & 90 & 69.50 & 5.50 & 0 & 80 & 86.88 & 6.88 & 0.72 \\
\hline Study_31 & 42 & 62.74 & 11.33 & 0 & 100 & 62.74 & 11.33 & 1.75 \\
\hline Study_32 & 50 & 4.87 & 1.15 & 0 & 5 & 97.40 & 23.00 & 3.25 \\
\hline Study_33 & 70 & 24.93 & 3.84 & 0 & 40 & 62.33 & 9.60 & 1.15 \\
\hline Study_34 & 34 & 27.60 & 11.30 & 0 & 50 & 55.20 & 22.60 & 3.88 \\
\hline Study_35 & 24 & 78.20 & 12.04 & 0 & 100 & 78.20 & 12.04 & 2.46 \\
\hline Study_36 & 28 & 85.71 & 5.70 & 0 & 100 & 85.71 & 5.70 & 1.08 \\
\hline Study_37 & 37 & 84.46 & 8.66 & 0 & 100 & 84.46 & 8.66 & 1.42 \\
\hline Study_38 & 42 & 59.21 & 6.28 & 0 & 100 & 59.21 & 6.28 & 0.97 \\
\hline Study_39 & 30 & 62.85 & 7.71 & 0 & 100 & 62.85 & 7.71 & 1.41 \\
\hline Study_40 & 36 & 78.34 & 6.17 & 0 & 100 & 78.34 & 6.17 & 1.03 \\
\hline Study_41 & 14 & 62.50 & 14.24 & 0 & 100 & 62.50 & 14.24 & 3.81 \\
\hline Study_42 & 42 & 62.74 & 11.33 & 0 & 100 & 62.74 & 11.33 & 1.75 \\
\hline
\end{tabular}


Table 3: Tabulation of Heterogeneity Test data

\begin{tabular}{|c|c|c|c|c|c|c|c|}
\hline Study & $E S_{i}$ & $S E_{i}$ & $w_{i}$ & $w_{i}^{2}$ & $w_{i} \cdot E S_{i}$ & $w_{i} \cdot E S_{i}^{2}$ & $\left(w_{i} \cdot E S_{i}\right)^{2}$ \\
\hline Study_1 & 85.04 & 0.96 & 1.085 & 1.177 & 92.274 & 7847.007 & 8514.547 \\
\hline Study_2 & 78.20 & 1.61 & 0.386 & 0.149 & 30.169 & 2359.184 & 910.144 \\
\hline Study_3 & 73.37 & 3.59 & 0.078 & 0.006 & 5.693 & 417.684 & 32.409 \\
\hline Study_4 & 81.38 & 1.21 & 0.683 & 0.467 & 55.584 & 4523.396 & 3089.540 \\
\hline Study_5 & 77.18 & 1.71 & 0.342 & 0.117 & 26.394 & 2037.123 & 696.667 \\
\hline Study_6 & 76.94 & 2.77 & 0.130 & 0.017 & 10.027 & 771.516 & 100.551 \\
\hline Study_7 & 82.61 & 1.43 & 0.489 & 0.239 & 40.398 & 3337.284 & 1632.004 \\
\hline Study_8 & 74.00 & 1.34 & 0.557 & 0.310 & 41.212 & 3049.677 & 1698.417 \\
\hline Study_9 & 84.60 & 0.36 & 7.716 & 59.537 & 652.778 & 55225.000 & 426118.827 \\
\hline Study_10 & 75.60 & 0.58 & 2.973 & 8.837 & 224.732 & 16989.774 & 50504.679 \\
\hline Study_11 & 85.55 & 2.43 & 0.169 & 0.029 & 14.488 & 1239.446 & 209.901 \\
\hline Study_12 & 68.30 & 2.33 & 0.184 & 0.034 & 12.581 & 859.270 & 158.277 \\
\hline Study_13 & 87.62 & 3.64 & 0.075 & 0.006 & 6.613 & 579.434 & 43.732 \\
\hline Study_14 & 83.61 & 0.99 & 1.020 & 1.041 & 85.308 & 7132.570 & 7277.390 \\
\hline Study_15 & 75.14 & 0.78 & 1.644 & 2.702 & 123.504 & 9280.111 & 15253.306 \\
\hline Study_16 & 82.42 & 1.25 & 0.640 & 0.410 & 52.749 & 4347.556 & 2782.436 \\
\hline Study_17 & 68.26 & 1.06 & 0.890 & 0.792 & 60.751 & 4146.874 & 3690.703 \\
\hline Study_18 & 75.34 & 0.48 & 4.340 & 18.838 & 326.997 & 24635.918 & 106926.729 \\
\hline Study_19 & 77.65 & 1.54 & 0.422 & 0.178 & 32.742 & 2542.386 & 1072.013 \\
\hline Study_20 & 83.61 & 1.55 & 0.416 & 0.173 & 34.801 & 2909.732 & 1211.127 \\
\hline Study_21 & 70.66 & 2.49 & 0.161 & 0.026 & 11.397 & 805.283 & 129.882 \\
\hline Study_22 & 76.45 & 1.32 & 0.574 & 0.329 & 43.876 & 3354.340 & 1925.126 \\
\hline Study_23 & 88.91 & 2.01 & 0.248 & 0.061 & 22.007 & 1956.632 & 484.303 \\
\hline Study_24 & 92.10 & 0.63 & 2.520 & 6.348 & 232.048 & 21371.655 & 53846.448 \\
\hline Study_25 & 70.40 & 3.12 & 0.103 & 0.011 & 7.232 & 509.139 & 52.303 \\
\hline Study_26 & 86.76 & 1.02 & 0.961 & 0.924 & 83.391 & 7235.003 & 6954.059 \\
\hline Study_27 & 83.48 & 1.68 & 0.354 & 0.126 & 29.578 & 2469.143 & 874.838 \\
\hline Study_28 & 79.36 & 0.97 & 1.063 & 1.130 & 84.345 & 6693.601 & 7114.041 \\
\hline Study_29 & 86.43 & 0.97 & 1.063 & 1.130 & 91.859 & 7939.361 & 8438.050 \\
\hline Study_30 & 86.88 & 0.72 & 1.929 & 3.721 & 167.593 & 14560.444 & 28087.277 \\
\hline Study_31 & 62.74 & 1.75 & 0.327 & 0.107 & 20.487 & 1285.325 & 419.698 \\
\hline Study_32 & 97.40 & 3.25 & 0.095 & 0.009 & 9.221 & 898.155 & 85.032 \\
\hline Study_33 & 62.33 & 1.15 & 0.756 & 0.572 & 47.130 & 2937.640 & 2221.278 \\
\hline Study_34 & 55.20 & 3.88 & 0.066 & 0.004 & 3.667 & 202.402 & 13.445 \\
\hline Study_35 & 78.20 & 2.46 & 0.165 & 0.027 & 12.922 & 1010.516 & 166.983 \\
\hline Study_36 & 85.71 & 1.08 & 0.857 & 0.735 & 73.483 & 6298.186 & 5399.679 \\
\hline Study_37 & 84.46 & 1.42 & 0.496 & 0.246 & 41.887 & 3537.736 & 1754.481 \\
\hline Study_38 & 59.21 & 0.97 & 1.063 & 1.130 & 62.929 & 3726.033 & 3960.073 \\
\hline Study_39 & 62.85 & 1.41 & 0.503 & 0.253 & 31.613 & 1986.883 & 999.388 \\
\hline Study_40 & 78.34 & 1.03 & 0.943 & 0.888 & 73.843 & 5784.858 & 5452.783 \\
\hline Study_41 & 62.50 & 3.81 & 0.069 & 0.005 & 4.306 & 269.098 & 18.538 \\
\hline Study_42 & 62.74 & 1.75 & 0.327 & 0.107 & 20.487 & 1285.325 & 419.698 \\
\hline Sum & 3249.53 & 70.5 & 38.88077 & 112.945 & 3105.093 & 250347.7 & 760740.8 \\
\hline
\end{tabular}


heterogeneity test using the following equation (Retnawati, Apino, et al., 2018).

$$
Q=\sum w_{i} E S_{i}^{2}-\frac{\left(\sum w_{i} E S_{i}\right)^{2}}{\sum w_{i}}
$$

With the criteria for decision making statistically, Ho rejected if the chi-square table. The Ho here states that the effect size $(\overline{E S})$ between homogeneous Studies.

For this purpose, the analysis is done by extending the data as presented in table 3 .

By using the values in table 3 , the values are obtained

$$
Q=\sum w_{i} E S_{i}{ }^{2}-\frac{\left(\sum w_{i} E S_{i}\right)^{2}}{\sum w_{i}}=250347.7-\frac{760740.8}{38.88}=230781.32
$$

Because the value is too large compared to the value of the chi-square table $\left(\mathcal{X}^{2}=56,94\right)$, then Ho is rejected so that it can be proved that the effect of size between heterogeneous studies. Because the effect size is heterogeneous, the fixed (fixed-effect model) can not be used. The recommended model is random (random effect model).

For analysis with a random model, first, estimate $C$ and know the square

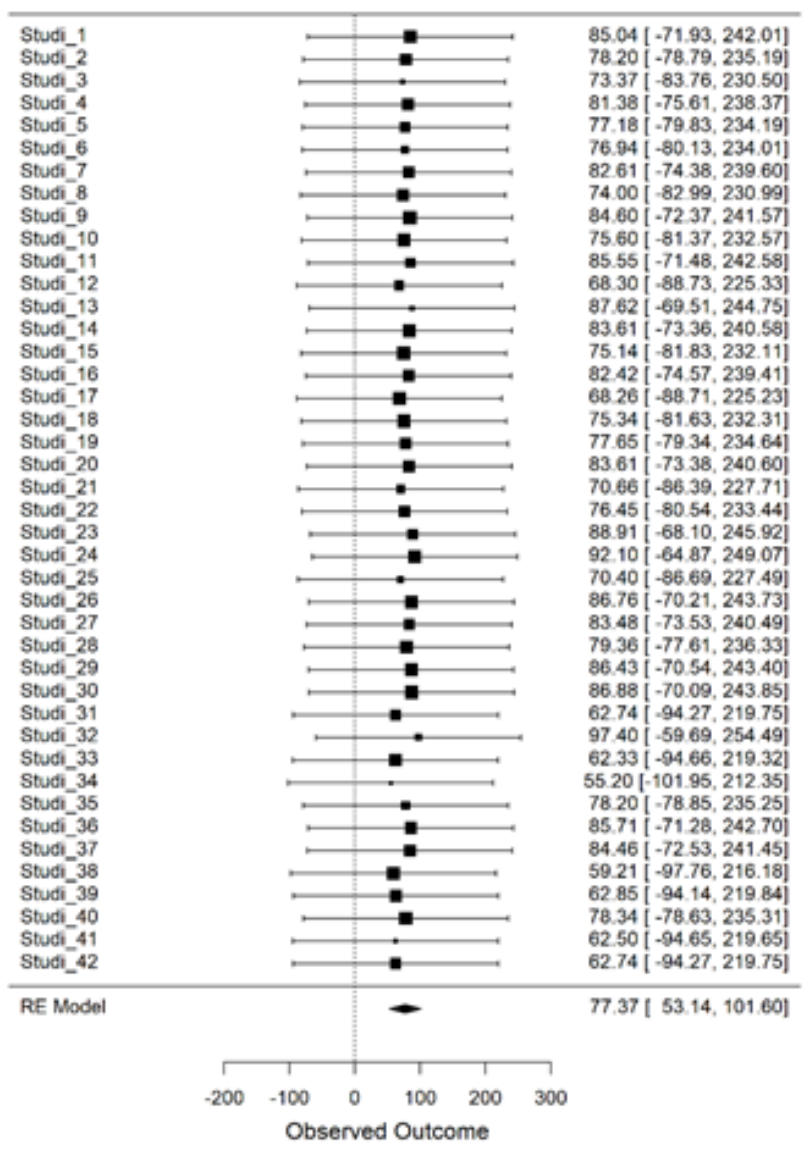

Figure 1: Forest Plot Summary Effect results with a random model

$$
\begin{aligned}
& C=\sum w_{i}-\frac{\sum w_{i}^{2}}{\sum w_{i}}=38.88-\frac{112.945}{38.88}=35.98 \\
& \tau^{2}=\frac{Q-d f}{C}=\frac{230781.32-41}{35.98}=6414.044
\end{aligned}
$$

By using tau squared $\left(\tau^{2}\right)$, the new variance and weighting can be determined. The complete calculation is presented in table 4 . The data in table 4 can be determined the value of the mean effect size of aggregation (effect size aggregates ) or summary effect or mean weighted at 77.37 with a standard error of the mean weighted random models for 12.36 . So that at the 95\% significance level, will obtain an upper and lower limit of 53.14 to 101.60 . In general, these values can also be seen from the forest plot, as presented in Figure 1. Based on the forest plot in Figure 1, the summary effect position appears to be almost the same or close to each study's effect size. These conditions show the consistency of forty-two studies' effect size greatly contributes to the value of the summary effect.

Moreover, the forest plot can also be viewed as relative weight studies towards the total. It can be seen from the box area for each study that shows each study's weight (Retnawati, 2014). It appears from the forest plot of figure 1, from fortytwo Studies obtained the highest relative weight of $2.382 \%$ of the total relative weight that is almost owned by more than $50 \%$ of the entire study. While the relative weight lower was owned by the 34 studies, which only amounted to $2.377 \%$ of the relative weight in total.

The next analysis calculates the value of $Z^{*}$ and tests the hypothesis related to the significance of the summary effect value. The values $Z$ * to test the null hypothesis $(\mathrm{H} 0$ : true effect $\theta=\mathbf{0}$ ) using the equation; with $p$-value one-tailed test: $p *=1-\Phi( \pm|Z *|)$ and $p$-value two-tailed test: $p *=$ $2[1-\Phi(|Z *|)]$, where $\Phi(|Z *|)$ standard cumulative normal distribution ( standard normal cumulative distribution ). $\Phi$ (| $Z * \mid)$ can be calculated with MS. Excel with the function "= NORMSDIST ( $Z^{*}$ )." By using this analysis, the value and value of $\Phi(|Z *|)=\Phi(6.26)=1$ will be obtained $p$-value one-tailed test: $p *=0.00$ and $p$-value two-tailed test: $p *=0$.

Based on the random-effect model calculation results, a summary effect of 77.37 is obtained with a $95 \%$ confidence interval ranging from 53.14 to 101.60 . While the results of testing the null hypothesis ( $\mathrm{H} 0$ : true effect $\theta=\mathbf{0}$ ) leads to a decision rejecting Ho. Because the value $Z$ of summary effect amounted to 77.37 with a p-value one-tailed test $(0,00)$ is smaller than the value of $\alpha(0.05)$ and the two-tailed test $(0$, $00) \mathrm{p}$-value is also smaller than $a(0.05)$. The conclusion $\mathrm{d}$ ap at drawn by the random-effect model is that innovative applied learning can improve student achievement HOTS.

Publication bias refers to the possibility of finding research that accepts a null hypothesis (absence of statistically significant effects) or negatives (the effect is significant but in the opposite direction to general or expected construction 
of the theory). Still, it tends to be unpublished compared to research results that show the effect positive (the impact is significant and by the general or expected construction of

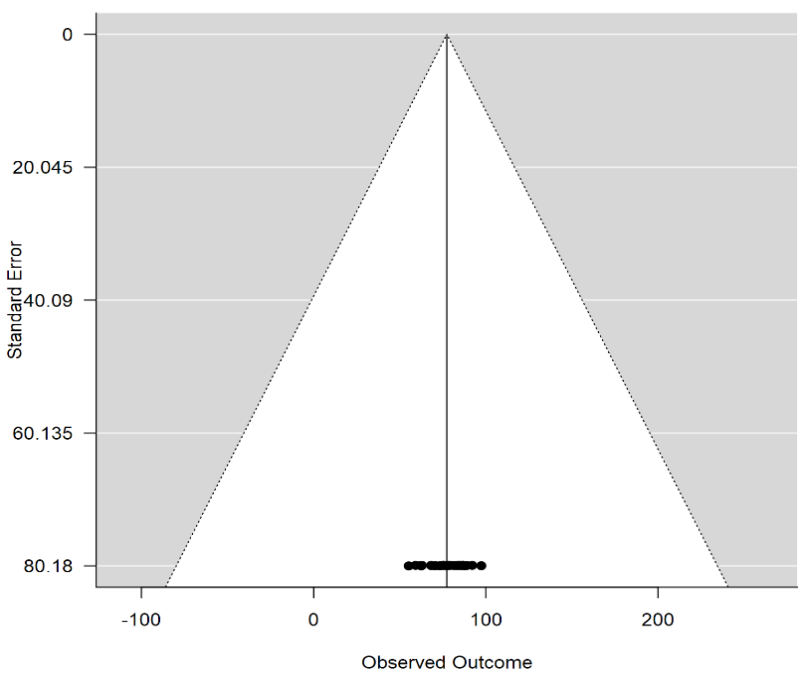

Figure 2: Funnel Plot

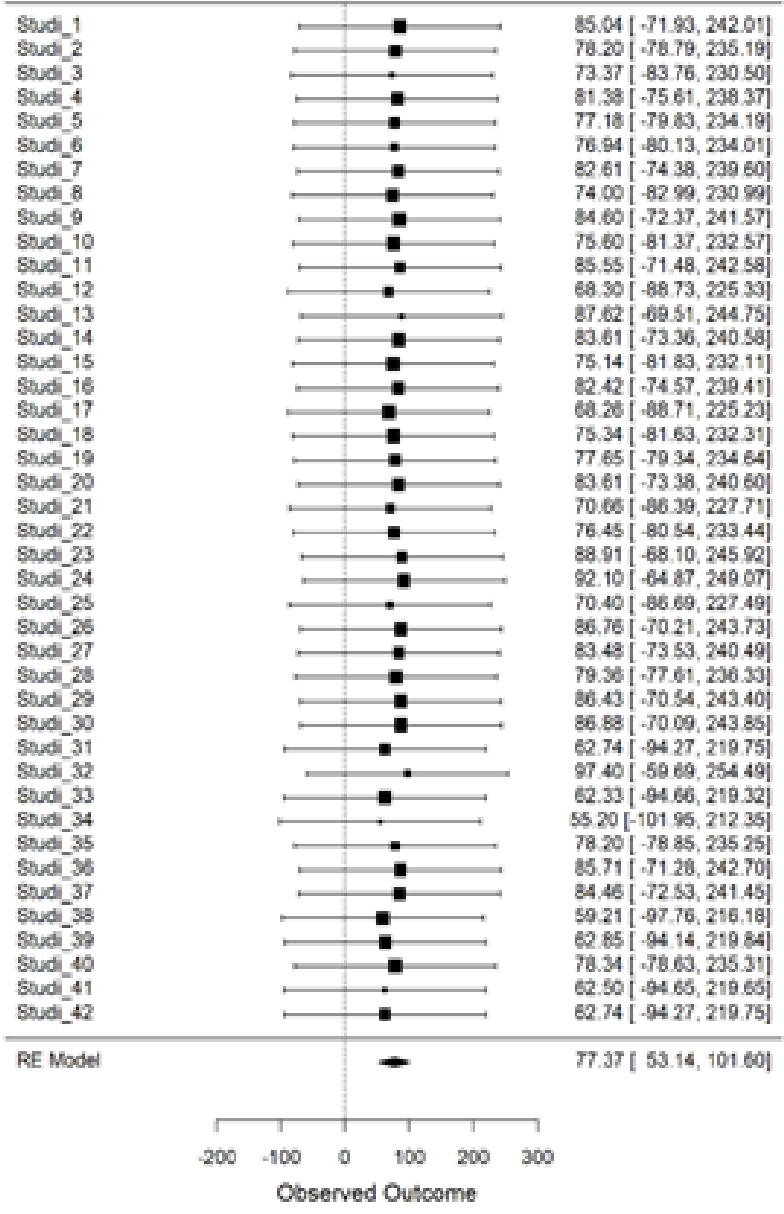

Figure 4: (a) Forest Plot Before and (b) Forest Plot After Using the Trim and Fill Method the theory). Studies with statistically significant results are more likely to be found in published literature than studies that report no statistically significant effects; about 61 to 68

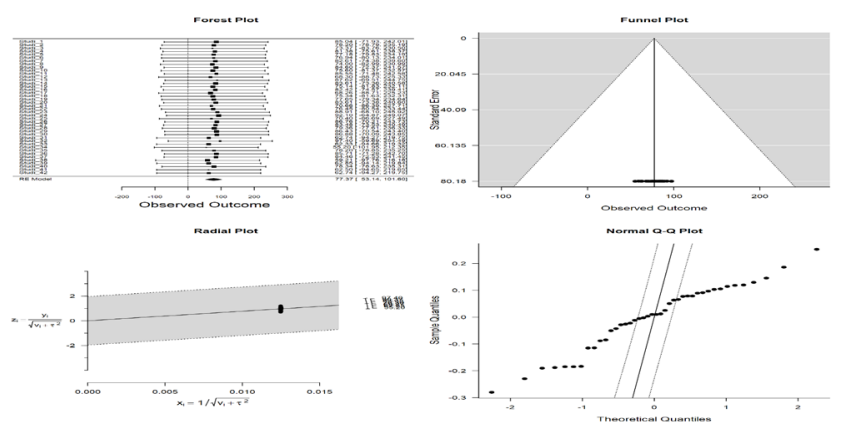

Figure 3: Funnel Plot with Trim and Fill methods

Table 3: Rank Correlation and Regression Results from Funnel Plot

\begin{tabular}{llll}
\hline \multicolumn{2}{l}{ Metode Rank Correlation } & \multicolumn{2}{l}{ Metode Regression } \\
\hline p-value & Rank Correlation & $p$-value & Regression Coeficient \\
\hline 0,109 & -0.186 & 0.852 & -0.186 \\
\hline
\end{tabular}

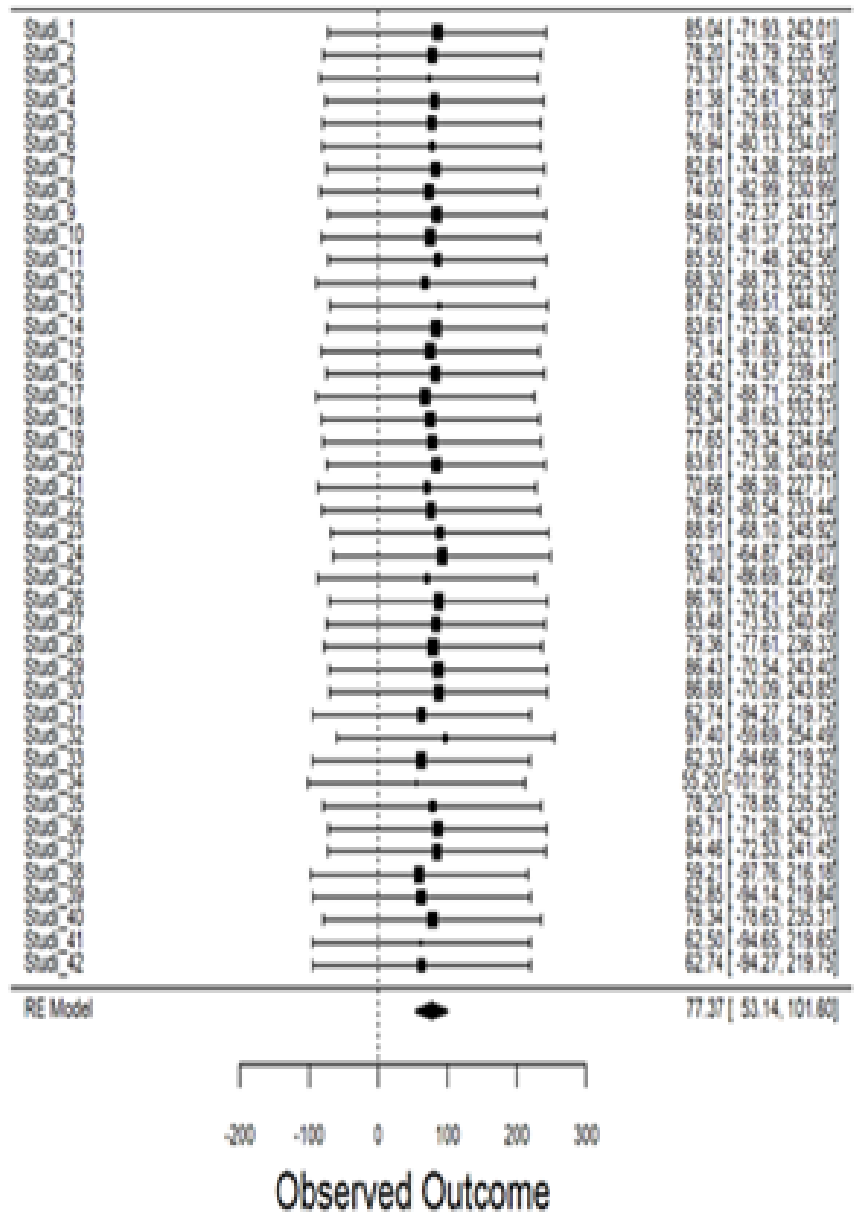


Table 4: New variance and weighting

\begin{tabular}{|c|c|c|c|c|c|c|}
\hline Study & $E S_{i}$ & $S E_{i}$ & $V^{*}$ & $S E^{*}$ & $w i^{*}$ & $w i^{*} . E S_{i}$ \\
\hline Study_1 & 85.04 & 0.96 & 6414.97 & 80.09 & 0.000156 & 0.013257 \\
\hline Study_2 & 78.20 & 1.61 & 6416.64 & 80.10 & 0.000156 & 0.012187 \\
\hline Study_3 & 73.37 & 3.59 & 6426.93 & 80.17 & 0.000156 & 0.011416 \\
\hline Study_4 & 81.38 & 1.21 & 6415.51 & 80.10 & 0.000156 & 0.012685 \\
\hline Study_5 & 77.18 & 1.71 & 6416.97 & 80.11 & 0.000156 & 0.012027 \\
\hline Study_6 & 76.94 & 2.77 & 6421.72 & 80.14 & 0.000156 & 0.011981 \\
\hline Study_7 & 82.61 & 1.43 & 6416.09 & 80.10 & 0.000156 & 0.012875 \\
\hline Study_8 & 74.00 & 1.34 & 6415.84 & 80.10 & 0.000156 & 0.011534 \\
\hline Study_9 & 84.60 & 0.36 & 6414.17 & 80.09 & 0.000156 & 0.01319 \\
\hline Study_10 & 75.60 & 0.58 & 6414.38 & 80.09 & 0.000156 & 0.011786 \\
\hline Study_11 & 85.55 & 2.43 & 6419.95 & 80.12 & 0.000156 & 0.013326 \\
\hline Study_12 & 68.30 & 2.33 & 6419.47 & 80.12 & 0.000156 & 0.01064 \\
\hline Study_13 & 87.62 & 3.64 & 6427.29 & 80.17 & 0.000156 & 0.013632 \\
\hline Study_14 & 83.61 & 0.99 & 6415.02 & 80.09 & 0.000156 & 0.013033 \\
\hline Study_15 & 75.14 & 0.78 & 6414.65 & 80.09 & 0.000156 & 0.011714 \\
\hline Study_16 & 82.42 & 1.25 & 6415.61 & 80.10 & 0.000156 & 0.012847 \\
\hline Study_17 & 68.26 & 1.06 & 6415.17 & 80.09 & 0.000156 & 0.01064 \\
\hline Study_18 & 75.34 & 0.48 & 6414.27 & 80.09 & 0.000156 & 0.011746 \\
\hline Study_19 & 77.65 & 1.54 & 6416.42 & 80.10 & 0.000156 & 0.012102 \\
\hline Study_20 & 83.61 & 1.55 & 6416.45 & 80.10 & 0.000156 & 0.013031 \\
\hline Study_21 & 70.66 & 2.49 & 6420.24 & 80.13 & 0.000156 & 0.011006 \\
\hline Study_22 & 76.45 & 1.32 & 6415.79 & 80.10 & 0.000156 & 0.011916 \\
\hline Study_23 & 88.91 & 2.01 & 6418.08 & 80.11 & 0.000156 & 0.013853 \\
\hline Study_24 & 92.10 & 0.63 & 6414.44 & 80.09 & 0.000156 & 0.014358 \\
\hline Study_25 & 70.40 & 3.12 & 6423.78 & 80.15 & 0.000156 & 0.010959 \\
\hline Study_26 & 86.76 & 1.02 & 6415.08 & 80.09 & 0.000156 & 0.013524 \\
\hline Study_27 & 83.48 & 1.68 & 6416.87 & 80.11 & 0.000156 & 0.013009 \\
\hline Study_28 & 79.36 & 0.97 & 6414.98 & 80.09 & 0.000156 & 0.012371 \\
\hline Study_29 & 86.43 & 0.97 & 6414.98 & 80.09 & 0.000156 & 0.013473 \\
\hline Study_30 & 86.88 & 0.72 & 6414.56 & 80.09 & 0.000156 & 0.013544 \\
\hline Study_31 & 62.74 & 1.75 & 6417.11 & 80.11 & 0.000156 & 0.009777 \\
\hline Study_32 & 97.40 & 3.25 & 6424.61 & 80.15 & 0.000156 & 0.01516 \\
\hline Study_33 & 62.33 & 1.15 & 6415.37 & 80.10 & 0.000156 & 0.009716 \\
\hline Study_34 & 55.20 & 3.88 & 6429.10 & 80.18 & 0.000156 & 0.008586 \\
\hline Study_35 & 78.20 & 2.46 & 6420.10 & 80.13 & 0.000156 & 0.012181 \\
\hline Study_36 & 85.71 & 1.08 & 6415.21 & 80.10 & 0.000156 & 0.01336 \\
\hline Study_37 & 84.46 & 1.42 & 6416.06 & 80.10 & 0.000156 & 0.013164 \\
\hline Study_38 & 59.21 & 0.97 & 6414.98 & 80.09 & 0.000156 & 0.00923 \\
\hline Study_39 & 62.85 & 1.41 & 6416.03 & 80.10 & 0.000156 & 0.009796 \\
\hline Study_40 & 78.34 & 1.03 & 6415.10 & 80.09 & 0.000156 & 0.012212 \\
\hline Study_41 & 62.50 & 3.81 & 6428.56 & 80.18 & 0.000156 & 0.009722 \\
\hline Study_42 & 62.74 & 1.75 & 6417.11 & 80.11 & 0.000156 & 0.009777 \\
\hline sum & 3086.29 & 67.93 & 256714.1 & 3204.46 & 0.006233 & 0.4809 \\
\hline
\end{tabular}


per cent are likely to occur (Dickersin et al., 1987). To detect the publication bias can be viewed from the Funnel Plot, Rank Correlation and Regression Method, and Trim and Fill.

\section{Funnel Plot}

If there is no publication bias, the research will be distributed symmetrically about the summary effect $(\mathrm{M})$ because the sampling error is random. Conversely, suppose there is a publication bias. In that case, the research will not follow the expected model ( asymmetric, some research is lost in the middle, and more research is lost at the bottom) (Retnawati, Apino, et al., 2018). The resulting output Jasp software obtained funnel plot for analysis of meta is presented in Figure 2.

Figure 2 is a funnel plot with a random model showing that the forty-two samples sampled in the meta-analysis are small sample sizes. If you pay close attention, in general, the whole study is symmetrically distributed. It can be interpreted that there is no potential for publication bias related to conclusions drawn.

\section{Rank Correlation and Regression Method}

The rank correlation and regression method is the development of statistical tests of the funnel plot. Output for Rank correlation and regression method presented in Tabel 3.

Table 3 shows that the p-value of the two methods ( rank correlation and regression ) is greater than the value of $a$ (0.05). It can be interpreted that the funnel plot formed by this random model is symmetrical, or in other words, there is no evidence of publication bias. Negative rank correlation $(-0.186)$ indicates that studies with large sample sizes are not included in the meta-analysis research sample, more dominant studies with small sample sizes. The regression coefficient is the coefficient of the estimated bias (-0.186). Overall it can be interpreted that we do not have enough strength to detect bias using rank correlation and regression methods.

\section{Trim and Fill}

Trim and Fill use an iterative procedure to remove the most extreme small research from the funnel plot's positive side, recalculating the effect size on each iteration until the funnel plot is symmetrical. In theory, this will produce an unbiased effect size estimate. In addition to this trim, the effect size is adjusted, reduces the effect variance, and results in a narrower confidence interval. Therefore, it is necessary to add back original research into the analysis. This fill has no impact on point estimation but improves variance (Duval \& Tweedie, 2000).

A computer program that can combine Trim and Fill ( Trim and Fill ) can create a funnel plot that includes research observed with missing research (not published). Researchers can see how the effect size shifts when missing (not published) studies are included in the analysis. If the change is small, other people can immediately trust our conclusions (the reported results are valid). The funnel plot of the Trim and Fill method for this study is presented in Figure 3. Figure 3 was obtained from the JASP software output version 0.11.1.0 using the Trim and Fill method. In Figure 3, there is no visible circle in the funnel plot of the random effect model. It can mean that there is no or not found missing (unpublished) research related to this study.

Apart from the funnel plot, it can also be viewed from the forest plot. If the research indicates publication bias, then the summary effect of the random-effect model will shift or drop from the previous summary effect ( summary effect before being analyzed by the Trim-Fill method), and the number of research samples will increase by itself (label: filled 1, filled $2, \ldots$ and filled $\mathrm{n}$ ). Based on this analysis, it can be seen that the conclusions regarding the application of innovative learning towards the achievement of students HOTS are free of potential publication bias. The forest plot's appearance also strengthens before and after using the Trim and Fill method, as presented in Figure 4. Figure 4 shows that the fixed-effect model's summary effect before using the Trim and Fill method (original) is the same as after being subjected to Trim and Fill's method. This condition indicates that the conclusions based on the random effect model on innovative learning in achieving HOTS students are valid.

\section{Discussion}

Based on the explanation of the study results, it can be obtained based on a meta-analysis that innovative learning has a strong influence on the achievement of HOTS students. This influence can be seen from the value of the summary effect or mean effect size with a random model of 77.37 and a $Z$ value of 6.26, which statistically proves that the effect is significant. This significant influence can not be separated from the applied innovative learning. A funnel plot with a random model shows that the forty-two samples sampled in a meta-analysis are small sample sizes and symmetrically distributed. It can be interpreted that there is no potential for publication bias related to the conclusions drawn. This condition is also reinforced by the forest plot's views, which showed the fixed-effect models' summary effect before using the Trim and Fill (original) together with a summary effect after having been subjected to method Trim and Fill. The conclusion that can be drawn based on the random-effect model is that innovative learning that is applied can improve HOTS students' achievement. This conclusion is free from publication bias, so it is valid (Ateş, 2021; Candra \& Retnawati, 2020)

Innovative learning is meant active learning and studentcentred learning that is deemed appropriate to training HOTS students (Akyol \& Garrison, 2011; Limbach \& Waugh, 2010; 
Retnawati, Djidu, et al., 2018). Active learning and studentcentered learning such as problem-based learning (PBL) (Mohd Zin Mokhtar et al., 2013), project-based learning (PjBL) (Vidergor \& Krupnik-Gottlieb, 2015)making meaning and transferring in very short times. Problem based learning and project based learning facilitate meaningful learning, development of complex skills and independent learner proficiency (For example: Tarhan 2007; Hmelo-Silver, 2004, learning discovery, and learning-based inquiry (Orlich et al., 2010), or other learning models that use contextual problems are some examples of strategies of innovative learning to train the right HOTS students. Besides, Protheroe (Goethals, 2013) and Miri, David, and Uri (Miri et al., 2007)within the framework of science education. Within a pre-, post-, and post-post experimental design, high school students, were divided into three research groups. The experimental group $(\mathrm{n}=57$ mentioned that group discussions and solving complex and interdisciplinary problems in the learning process were also important activities to train HOTS students. Innovative learning is very thick with this activity (Lehmann et al., 2008) where their boundaries are often difficult to identify, and where societal rather than technical issues play increasingly bigger roles, problems cannot be solved by applying a technical solution alone. It thus becomes important for engineers to be skilled not only in terms of their particular technical field but also their ability to identify non-technical aspects of problems, the interaction between these aspects and possible solutions. Introducing and integrating these aspects into engineering education is certainly not an easy task and requires innovative approaches. In this article, focus is placed on the so-called Aalborg Model, a problem-oriented and project-based learning paradigm utilised at Aalborg University (Denmark.

This study's results can also provide empirical evidence and contribute to alternative learning consistent with that recommended in implementing the 2013 curriculum. As is well known, one of the components that become a curriculum review is high-level thinking skills (HOTS). HOTS is an essential element for solving new problems in the 21st Century (Brookhart, 2010; Moseley et al., 2005; Thompson, 2008). HOTS also plays an essential role in applying, connecting, or manipulating prior knowledge to effectively solve new problems (Thomas \& Thorne, 2009). In line with this, there are two main reasons HOTS is important for students: students must succeed at school and make a positive contribution to society (Conklin, 2012). Therefore, HOTS is very important in the learning process so that students can contribute to the community. In the revised Bloom taxonomy, HOTS is defined as a slice between the top three levels of ability in the cognitive dimension (analyzing, evaluating, creating) and three levels of the dimension of knowledge (conceptual, procedural, metacognitive) (Anderson \& Krathwohl, 2001; Thompson, 2008). Besides, creative thinking and critical thinking skills are included in higher-order thinking skills (HOTS) (Miri et al., 2007; Moseley et al., 2005)within the framework of science education. Within a pre-, post-, and post-post experimental design, high school students, were divided into three research groups. The experimental group $(\mathrm{n}=57$.

HOTS's importance is no exception for students in school, making every education component work together to practice students' thinking ability. It means that familiarizing students with HOTS activities is essential to help them solve new problems, adjust to new situations, and make decisions about specific issues (Indiani \& Retnawati, 2017).

In practical reviews, with innovative learning, for example, problem-based learning, students become more challenged to explore various possible ideas that can be used to solve problems (Ezi Apino \& Retnawati, 2016; Jailani \& Retnawati, 2016)which used pretest-posttest experimental non-equivalent control group. Experimental class was a class which was taught by using problem-based learning, while the control class was a class which was taught by using direct instruction. The population of this research was the seventh graders of several Junior High Schools in DI Yogyakarta which have implemented problem based learning. The samples of this research were 515 students of Junior High School students from 10 schools in four districts and one city in Yogyakarta. The schools from which the samples were taken were both public and private schools which were selected based on their achievement in the national examination. The schools were selected using stratified random sampling, while the classes were selected randomly. The quantitative data analysis was conducted by using both descriptive and inferential statistic. The results showed that: (1. Loewen (Loewen, 1995) suggested that giving contextual creative problems and placing students dominant in learning can produce students' awareness that not all issues have only one correct solution. It can trigger and train students' creativity in learning, and creativity is part of HOTS. Another factor that causes an increase in students' HOTS is that innovative learning tends to lead to meaningful learning activities. Students are actively involved in the discussion process to build knowledge and utilize various relevant sources to explore the desired knowledge. These findings are consistent with Bohan \& Bohan (Bohan \& Bohan, 2020) that the learning process involving students' active participation to solve various problems can present meaningful learning activities for students and enhance students' higher-order performance thinking skills.

The results are relevant to previous studies. The learning to improve or train HOTS students can be done with several activities, such as involving students in problem-solving activities, providing opportunities for students to build their knowledge and improve their abilities, the ability to analyze, evaluating, and creating (Apino \& Retnawati, 2017), involves students undergoing group discussions and communicating 
the results of problem-solving through presentations (Djidu \& Jailani, 2016). In other words, building a learning-oriented HOTS can be done by minimizing teachers' dominance and maximizing the role of students in the learning process. Learning like this corresponds to innovative learning. There are at least three strategies carried out by teachers in implementing innovative learning, first in planning activities, namely teachers preparing physically and mentally, preparing HOTS-based lesson plans, preparing media, and appropriate evaluations. Second, in core learning, the teacher conducts learning using innovative learning steps, such as problembased learning, starting from presenting problems, such as how to save fuel, organizing learning, directing constructively how to save fuel, presenting the results of discussions, and concluding. Third, closing the lesson by making conclusions together and evaluating with HOTS-based questions covering aspects of attitudes, knowledge, and skills (Inayati, 2020) which is required learning model with high level-thingking or commonly known as HOTS (Higher Order Thinking Skills.

Besides $\mathrm{n}$ the context of increasing students' HOTS achievements, this innovative learning is also expected to minimize and even eliminate student difficulties in solving HOTS problems. It is important because conditions in the field have not fully implemented innovative learning. As a result, HOTS students' achievement is still low, marked by students' difficulties when faced with questions that measure HOTS. Research by Hadi, Retnawati, Munadi, Apino, \& Wulandari (Hadi et al., 2018) mentioned that the most common problem for students in completing test questions that measure HOTS is mathematical process skills. This difficulty is demonstrated by errors in implementing formulas, mathematical calculations errors, and errors in algebraic operations and manipulation. This condition suggests that one alternative that can be applied is innovative learning that facilitates students to optimize their thinking skills, including HOTS students.

\section{Conclusion}

This meta-analysis study investigates the effect size of innovative learnings on achieving student HOTS. It was found that the innovative learning applied had an effect on the achievement of students' HOTS and there was no potential for publication bias regarding the conclusions drawn. So that the conclusions made based on the random effect model about the application of innovative learning affect the achievement of students' HOTS are valid. These results indicate that innovative learning can be used as learning carried out in the classroom because it is empirically able to facilitate the achievement of students' HOTS.

\section{Limitation and Suggestion}

This meta-analysis study also has limitations in combining research results related to innovative learning in students' achievement of HOTS based on the mean value. First, the data collection used is limited to data whose mean values are explicitly stated, even though there may still be many research results that have not been included which are considered relevant. Second, in determining the research results (study) used as data, they did not consider the characteristics of the students (sample) who were in the study. In future research, it is hoped that the studies used in this meta-analysis will consider more studies and consider the characteristics of students (samples) so that the meta-analysis results are more valid.

\section{References}

Agustina, N., Imamora, M., \& Chandra, A. N. (2020). Pengembangan Modul Berbasis Pembelajaran CTL Untuk Mencapai HOTS Pada Materi Getaran Harmonis. Proceeding IAIN Batusangkar, 1(3), 169-176.

Akyol, Z., \& Garrison, D. R. (2011). Understanding cognitive presence in an online and blended community of inquiry: Assessing outcomes and processes for deep approaches to learning. British Journal of Educational Technology, 42(2), 233-250. https://doi. org/10.1111/j.1467-8535.2009.01029.x

Alandia, R. G., \& Suparwoto, S. (2019). Pengembangan Perangkat Pembelajaran Fisika Model Problem-Based Learning Berbantuan Web untuk Meningkatkan Kemampuan Berpikir Kritis dan Keterampilan Proses Sains Peserta Didik SMA. In S2 thesis, Program Pascasarjana. http://eprints.uny.ac.id/id/ eprint/67333

Aljarrah, K., \& Khataybeh, A. (2021). Representing teaching staff in Jordanian universities for the 21st century skills. Pegem Journal of Education and Instruction, 11(3), 17-25.

Ambarita, R. A., Yunastiti, Y., \& Indriayu, M. (2019). The Application of Group Investigation Based on Hands on Activities to Improve Learning Outcomes Based on Higher Order Thinking Skills of Students at SMA Negeri 2 Pematangsiantar. Budapest International Research and Critics in Linguistics and Education (BirLE) Journal, 2(2), 351-359. https://doi.org/10.33258/birle. $\mathrm{v} 2 \mathrm{i} 2.310$

Anazifa, R. D., \& Djukri. (2017). Project- based learning and problembased learning: Are they effective to improve student's thinking skills? Jurnal Pendidikan IPA Indonesia, 6(2), 346-355. https:// doi.org/10.15294/jpii.v6i2.11100

Anderson, L. W., \& Krathwohl, D. R. (2001). A revision of Bloom's taxonomy of educational objectives. A Taxonomy for Learning, Teaching and Assessing. Longman, New York.

Apino, E., \& Retnawati, H. (2017). Developing Instructional Design to Improve Mathematical Higher Order Thinking Skills of Students. Journal of Physics: Conference Series, 812(1), 12100. https://doi.org/10.1088/1742-6596/812/1/012100

Apino, Ezi, \& Retnawati, H. (2016). Creative Problem Solving to Improve Students' Higher Order Thinking Skills in Mathematics Instructions. Proceeding of $3 R d$ International Conference on Research, Implementation and Education of Mathematics and Science, May, 339-346.

Arends, R. I., \& Kilcher, A. (2010). Teaching for student learning: Becoming an accomplished teacher. In Teaching for Student 
Learning: Becoming an Accomplished Teacher. Routledge. https://doi.org/10.4324/9780203866771

Ariansyah, D., \& Soenarto, S. (2019). Pengaruh Pendekatan Contextual Teaching and Learning (CTL) Terhadap Kemampuan Berpikir Kritis Siswa pada Materi Rangkaian RLC. In S2 thesis, Program Pascasarjana. http://eprints.uny.ac.id/id/eprint/65975

Arisandi, G., \& Sutrisno, H. (2019). Penerapan Pembelajaran Kimia Berbasis Analogi untuk Menganalis Keterampilan Berpikir Tingkat Tinggi Siswa pada Materi Ikatan Kimia. In S2 thesis, Program Pascasarjana. http://eprints.uny.ac.id/id/eprint/66753

Ateş, A. (2021). The Relationship Between Parental Involvement in Education and Academic Achievement: A Meta-analysis Study. Pegem Egitim ve Ogretim Dergisi, 11(3), 50-66.

Azis Nur, A. (2016). Pengaruh Model Problem Based Learning Terhadap Keterampilan Berpikir Tingkat Tinggi dan hasil Belajar Biologi Siswa SMK-PP Negeri REA Timur Kabupaten Polewali mandar.

Baskoro, I. (2019). Efektivitas Pembelajaran Matematika Berbasis Teori Variasi Ditinjau Dari Keterampilan Berpikir Tingkat Tinggi Siswa Smk.S2 Thesis. Sekolah Pascasarjanan Universitas Negeri Yogyakarta Indonesia.

Bohan, H., \& Bohan, S. (2020). Extending the Regular Curriculum Through Creative Problem Solving. The Arithmetic Teacher, 41(2), 83-87. https://doi.org/10.5951/at.41.2.0083

Brookhart, S. M. (2010). How to assess higher-order thinking skills in your classroom. ASCD.

Candra, \& Retnawati, H. (2020). A meta-analysis of constructivism learning implementation towards the learning outcomes on civic education lesson. International Journal of Instruction, 13(2), 835-846. https://doi.org/10.29333/iji.2020.13256a

Çoğaltay, N., \& Karadağ, E. (2015). Introduction to meta-analysis. In Leadership and organizational outcomes (pp. 19-28). Springer.

Conklin, W. (2012). Higher-Order Thinking Skills to Develop 21st Century Learners. In Shell Education. Teacher Created Materials.

Crumb, L. N. (1983). Taxonomy Of Educational Objectives The Classification of Educational Goals. In Cataloging and Classification Quarterly (Vol. 3, Issue 1). Ann Arbor.

Dasilva, B. E., \& Suparno, S. (2019). Pengembangan Interactive Physics Mobile Learning Media Berbasis Android dengan Pendekatan Pembelajaran Scaffolding Pada Materi Termodinamika untuk Meningkatkan Higher Order Thinking Skill dan Self Efficacy Peserta Didik SMA. In S2 thesis, Program Pascasarjana. http:// eprints.uny.ac.id/id/eprint/65804

Dickersin, K., Chan, S., Chalmersx, T. C., Sacks, H. S., \& Smith, H. (1987). Publication bias and clinical trials. Controlled Clinical Trials, 8(4), 343-353. https://doi.org/10.1016/01972456(87)90155-3

Djidu, H., \& Jailani. (2016). Aktivitas Pembelajaran Matematika yang Dapat Melatih Kemampuan Berpikir Tingkat Tinggi Siswa. Seminar Nasional Matematika X Universitas Negeri Semarang, 314.

Duval, S., \& Tweedie, R. (2000). Trim and fill: A simple funnelplot-based method of testing and adjusting for publication bias in meta-analysis. Biometrics, 56(2), 455-463. https://doi. org/10.1111/j.0006-341X.2000.00455.x

Erny, Haji, S., \& Widada, W. (2017). Pengaruh pendekatan saintifik pada pembelajaran matematika terhadap kemampuan pemecahan masalah dan kemampuan berpikir tingkat tinggi siswa kelas X ipa sma negeri 1 kepahiang. Jurnal Pendidikan Matematika Raflesia, 6(7), 84-95.

Fauziah, S. (2013). The Effectiveness of PBL Online on Physics Students' Creativity and Critical Thinking: A Case Study at Universiti Malaysia Sabah. International Journal of Education and Research, 1(3), 1-18.

Ferty, Z. N., \& Suparno, S. (2019). Pengembangan Interactive Physics Mobile Learning Media Berbasis Android dengan Pendekatan Scaffolding untuk Meningkatkan HOTS dan Sikap Ilmiah Siswa SMA.

Fisher, R. (2010). Thinking Skill Strategies.pdf. In D. J. Arthur \& T. Cremin (Eds.), Learning to Teach in The Primary School (2nd ed., pp. 374-387). Routledge.

Folly Eldy, E., \& Sulaiman, F. (2013). Integrated PBL Approach: Preliminary Findings towards Physics Students' Critical Thinking and Creative-Critical Thinking. International Journal of Humanities and Social Science Invention ISSN (Online, 2(3), 2319-7722.

Freeman, P. R., Hedges, L. V., \& Olkin, I. (1986). Statistical Methods for Meta-Analysis. In Biometrics (Vol. 42, Issue 2). Academic press. https://doi.org/10.2307/2531069

Goethals, P. L. (2013). The Pursuit of Higher-Order Thinking in the Mathematics Classroom. United States Military Academy, West Point, NY.

Guedri, Z. (2001). Problem-based Learning: Bringing Higher Order Thinking to Business Schools. Cahier de Recherche OIPG No, 2, 002. http://neumann.hec.ca/oipg/fichiers/2001-002_-_ Problem-Based_Learning....pdf

Hadi, S., Retnawati, H., Munadi, S., Apino, E., \& Wulandari, N. F. (2018). The difficulties of high school students in solving higher-order thinking skills problems. Problems of Education in the 21st Century, 76(4), 520.

Haryanto, P. C., \& Arty, I. S. (2019). Pengembangan Video animasi IPA Berbasis Contextual Teaching And Learning (CTL) Untuk Meningkatkan High Order Thinking Skills (HOTS. In Dan Self Efficacy Peserta Didik SMP. S2 thesis, Program Pascasarjana. http://eprints.uny.ac.id/id/eprint/66366

Hendryarto, J. (2013). Penerapan Model Pembelajaran Inkuiri Untuk Melatih Kemampuan Berpikir Tingkat Tinggi Siswa Pada Materi Pokok Laju Reaksi (Implementation Inquiry Learning Model For Training High Order Thinking Skills Of The Students On Main Material Of Reaction Rate. UNESA Journal of Chemical Education, 2(2).

Hidayati, A. U., \& Retnawati, H. (2018). Keefektifan pendekatan PBL dan pendekatan saintifik ditinjau dari HOTS dan karakter. Jurnal Pendidikan Matematika Dan Sains, 6(1), $70-82$.

Inayati, U. (2020). Strategi Guru Dalam Menerapkan Pembelajaran HOTS Menggunakan Model Problem Based Learning. Auladuna : Jurnal Prodi Pendidikan Guru Madrasah Ibtidaiyah, 2(2), 27-34. https://doi.org/10.36835/au.v2i2.410

Indiani, V., \& Retnawati, H. (2017). Need Assessment To Develop Teaching Set For Improving Higher Order Thinking Skills of Junior High School Students In Learning Mathematics. Proceeding the International Conference on Education Innovation, 1(1), 848-853.

Jailani, J., Sugiman, S., \& Apino, E. (2017). Implementing the problem-based learning in order to improve the students 
HOTS and characters. Jurnal Riset Pendidikan Matematika, 4(2), 247. https://doi.org/10.21831/jrpm.v4i2.17674

Jailani, \& Retnawati, H. (2016). Keefektifan Pemanfaatan Perangkat Pembelajaran Berbasis Masalah untuk Meningkatkan HOTS dan Karakter Siswa. Jurnal Pendidikan Dan Pembelajaran, 23(2), 111-123. http://journal.um.ac.id/index.php/pendidikandan-pembelajaran/article/view/10162/4849

Khoiriah, \& Jalmo, T. (2020). Student Worksheet Based on Discovery Learning Combine With Assessment for Learning Higher Order Thinking Skills (AfL-HOTS) to Fostering High Level Thinking Skills of Students. The Online Journal of New Horizons in Education, 10(1), 69-77.

King, F. J., Goodson, L., \& Rohani, F. (2010). Higher order thinking skills: Definition, Teaching Strategies, Assessment. http://goo. gl/su233T.

Koza Çiftçi, \& Yıldız, P. (2019). The effect of gender on algebra achievement: The meta-analysis of trends in international mathematics and science study (TIMSS). Turkish Journal of Computer and Mathematics Education, 10(3), 617-627. https:// doi.org/10.16949/turkbilmat.568545

Krulik, S., \& Rudnick, J. A. (1999). Innovative tasks to improve critical and creative thinking skills. In D. L. V Stiff \& F. R. Curcio (Eds.), Developing Mathematical Reasong in Grades K-12 (pp. 138-145). NCTM.

Kurnia, I. A., \& Retnowati, E. (2019). Efektivitas Erroneous Worked Example dan Strategi Pengelompokan pada Pembelajaran Aritmetika Sosial Ditinjau dari Kemampuan Berpikir Tingkat Tinggi. In 22 thesis, Program Pascasarjana. http://eprints.uny. ac.id/id/eprint/66457

Lehmann, M., Christensen, P., Du, X., \& Thrane, M. (2008). Problemoriented and project-based learning (POPBL) as an innovative learning strategy for sustainable development in engineering education. European Journal of Engineering Education, 33(3), 283-295. https://doi.org/10.1080/03043790802088566

Limbach, B., \& Waugh, W. (2010). Developing higher level thinking. Journal of Instructional Pedagogies, 3, 9. https://aabri.com/ manuscripts/09423.pdf

Liu, S., Yang, X., Zhang, H., Wang, Y., Yoneda, T., \& Li, Z. (2018). Study on teaching methods for developing higher order thinking skills for college students in flipping classroom. Proceedings - 6th International Conference of Educational Innovation Through Technology, EITT 2017, 2018-March, 254-257. https://doi.org/10.1109/EITT.2017.69

Liu, X. (2014). Essentials of Science Classroom Assessment. In Essentials of Science Classroom Assessment. Sage Publications. https://doi.org/10.4135/9781483349442

Loewen, A. C. (1995). Creative problem solving. Teaching Children Mathematics, 2(2), 96-99.

Luthfiyah, R. A., Chrisnawati, H. E., Pramesti, G., \& Kuswardi, Y. (2019). Development of teaching materials to improve the student's high order thinking skills. AIP Conference Proceedings, 2194(1), 20057. https://doi.org/10.1063/ 1.5139789

Maghfiroh, L., \& Mulyani, E. (2019). Pengembangan Modul Pembelajaran Ekonomi dengan Pendekatan Problem Based Learning yang Berorientasi pada High Order Thinking Skills untuk Meningkatkan Kemampuan Berpikir Kritis dan Kreatif Peserta Didik. In S2 thesis, Program Pascasarjana. http:// eprints.uny.ac.id/id/eprint/65780
Malik, A., Setiawan, A., Suhandi, A., \& Permanasari, A. (2017). Enhancing pre-service physics teachers' creative thinking skills through HOT lab design. AIP Conference Proceedings, 1868(1), 70001. https://doi.org/10.1063/1.4995177

Mayasari, R., \& Adawiyah, R. (2016). Pengaruh Model Pembelajaran Berdasarkan Masalah Pada Pembelajaran Biologi Terhadap Hasil Belajar Dan Keterampilan Berpikir Tingkat Tinggi Di Sma. Jurnal Pendidikan Biologi Indonesia, 1(3). https://doi. org/10.22219/jpbi.vli3.2658

Miri, B., David, B. C., \& Uri, Z. (2007). Purposely teaching for the promotion of higher-order thinking skills: A case of critical thinking. Research in Science Education, 37(4), 353-369. https:// doi.org/10.1007/s11165-006-9029-2

Mohd Zin Mokhtar, Rohani Ahmad Tarmizi, Ahmad Fauzi Mohd Ayub, \& Nawawi, M. D. H. (2013). Motivation and Performance In Learning Calculus Through Problem-Based Learning. International Journal of Asian Social Science, 3(9), 1999-2005. http://www.aessweb.com/journal-detail. php?id=5007\%0AMOTIVATION

Moseley, D., Elliott, J., Gregson, M., \& Higgins, S. (2005). Thinking skills frameworks for use in education and training. British Educational Research Journal, 31(3), 367-390. https://doi. org/10.1080/01411920500082219

Najib, A. (2015). Pengaruh Penggunaan Program Simulasi PhET dalam Pembelajaran Inkuiri Laboratorium terhadap Penguasaan Konsep dan Keterampilan Berpikir Tingkat Tinggi. https://lib.unnes.ac.id/22920/

Nyoman Setiawan, I. G. A. (2015). Pengembangan Model Asesmen Autentik Pembelajaran Ipa Kontekstual Terintegrasi Dengan Model Pengajaran Berpikir Tingkat Tinggi Sebagai Upaya Meningkatkan Kompetensi Siswa Smp. JPI (Jurnal Pendidikan Indonesia), 1(1). https://doi.org/10.23887/jpi-undiksha. v1i1.4482

Orlich, D. C., Harder, R. J., Callahan, R. C., Trevisan, M. S., \& Brown, A. H. (2010). Teaching Strategies-Guide to Effective Instruction. Wadsworth Engage Learning.

Pigott, T. (2012). Advances in meta-analysis. Springer Science \& Business Media.

Presseisen, B. Z. (1988). Thinking skills: Meanings and models. In D. A. L. Costa (Ed.), Developing minds: A resource book for teaching thinking (pp. 43-48). ASCD.

Puspaningtyas, N. A. (2019). Peningkatan Higher Order Thinking Skills (HOTS) Melalui Strategi Pembelajaran Peningkatan Kemampuan Berpikir (SPPKB). In Jurnal Pendidikan dan Ekonomi, (Vol. 8, Issue 2). Sekolah Pascasarjana Universitas Negeri Yogyakarta Indonesia. http://e-journal.uajy. ac.id/14649/1/JURNAL.pdf

Putri, R., \& Ghufron, A. (2019). Efektivitas Strategi The Power of Two Terhadap Kecakapan Critical Thinking Siswa. In Jurnal Kependidikan: Penelitian Inovasi Pembelajaran (Vol. 3, Issue 2). Sekolah Pascasarjanan Universitas Negeri Yogyakarta Indonesia.

Rahayu, A., \& Utaminingsih, R. (2017). Efektivitas Penggunaan Teknik Effective Questioning Pada Mata Kuliah Ipa 1 Untuk Meningkatkan Keterampilan Berpikir Tingkat Tinggi Mahasiswa. In SOSIOHUMANIORA: Jurnal Ilmiah Ilmu Sosial dan Humaniora (Vol. 2, Issue 1). Jurnal Ilmiah Ilmu Sosial Dan Humaniora. https://doi.org/10.30738/sosio. v2i1.491 
Ramadhan, R. H. (2019). Pengembangan Komik Fisika Berbasis Kearifan Lokal Long Bumbung Berbantu Android Untuk Meningkatkan Kemampuan Representasi Fisis Dan HOTS Peserta Didik SMA Pada Materi Bunyi (Doctoral dissertation, Tesis. Sekolah Pascasarjanan Universitas Negeri Yogyakarta Indonesia.

Redhana, I. W. (2013). Model Pembelajaran Berbasis Masalah Dan Pertanyaan Socratik Untuk Meningkatkan Keterampilan Berpikir Kritis Siswa. In Jurnal Cakrawala Pendidikan (Issue 3, p. 3). https://doi.org/10.21831/cp.v0i3.1136

Retnawati, H., Apino, E., Kartianom, Djidu, H., \& Anazifa, R. D. (2018). Pengantar Analisis Meta (Issue August 2019). Parama Publishing.

Retnawati, H., Djidu, H., Kartianom, Apino, E., \& Anazifa, R. D. (2018). Teachers' knowledge about higher-order thinking skills and its learning strategy. Problems of Education in the 21st Century, 76(2), 215-230. https://doi.org/10.33225/pec/18.76.215

Rochani, S. (2016). Keefektifan pembelajaran matematika berbasis masalah dan penemuan terbimbing ditinjau dari hasil belajar kognitif kemampuan berpikir kreatif. Jurnal Riset Pendidikan Matematika, 3(2), 273. https://doi.org/10.21831/jrpm.v3i2.5722

Rosida, R., Fadiawati, N., \& Jalmo, T. (2017). Efektivitas Penggunaan Bahan Ajar E-Book Interaktif Dalam Menumbuhkan Keterampilan Berpikir Kritis Siswa. Jurnal Pembelajaran Fisika Universitas Lampung, 5(1), 116746.

Salam, A., \& Miriam, S. (2016). Pembelajaran Berbasis Learner Autonomy Untuk Melatihkan Kemampuan Berpikir Tingkat Tinggi Mahasiswa. In Mengembangkan Keterampilan Berpikir Tingkat Tinggi Melalui Pembelajaran IPA (pp. 53-60). Fakulatas Keguruan dan Ilmu Pendidikan Universitas Lambung Mangkurat. http://www.s2ipa.unlam.ac.id/category/ publikasi-ilmiah/proceeding/seminar-nasional-pendidikanipa-2016

Saputri, A. C., Sajidan, Rinanto, Y., Afandi, \& Prasetyanti, N. M. (2019). Improving students' critical thinking skills in cellmetabolism learning using Stimulating Higher Order Thinking Skills model. International Journal of Instruction, 12(1), 327-342. https://doi.org/10.29333/iji.2019.12122a

Saregar, A., Latifah, S., \& Sari, M. (2016). Efektivitas Model Pembelajaran CUPs: Dampak Terhadap Kemampuan Berpikir Tingkat Tinggi Peserta Didik Madrasah Aliyah Mathla’ul Anwar Gisting Lampung. Jurnal Ilmiah Pendidikan Fisika Al-Biruni, 5(2), 233-244. https://doi.org/10.24042/jpifalbiruni.v5i2.123

Sekarini, R. P. (2019). Pengembangan perangkat pembelajaran Science Outdoor Learning Berbasis Kontekstual Untuk Meningkatkan Curiosity dan High Order Thingking Skill (HOTS) Peserta Didik SMP. Sekolah Pascasarjanan Universitas Negeri Yogyakarta Indonesia.

Silva, E. (2009). Measuring skills for 21st-century learning. Phi Delta Kappan, 90(9), 630-634. https://doi.org/10.1177/ 003172170909000905

Smart, J. B., \& Marshall, J. C. (2013). Interactions Between Classroom Discourse, Teacher Questioning, and Student Cognitive Engagement in Middle School Science. Journal of Science Teacher Education, 24(2), 249-267. https://doi.org/10.1007/ s10972-012-9297-9

Stauffer, B. (2018). What are the 4 C's of 21 st century skills? In Applied Education System (pp. 1-9). https://www. aeseducation.com/blog/four-cs-21st-century-skills\%0Ahttps:// www.aeseducation.com/careercenter21/what-are-the-4-cs-of21st-century-skills

Suarsana, I. M. (2013). Pengembangan E-Modul Berorientasi Pemecahan Masalah Untuk Meningkatkan Keterampilan Berpikir Kritis Mahasiswa. JPI (Jurnal Pendidikan Indonesia), 2(2). https://doi.org/10.23887/jpi-undiksha.v2i2.2171

Suleman, M., \& Sugijarto, K. H. (2019). Pengembangan Media Visualisasi Tiga Dimensi 3D dengan Virtual Reality (3D-VR) Materi Laju Reaksi Terhadap Kemampuan Keterampilan Proses Sains dan Berpikir Kritis Pesrta Didik SMA. In S2 thesis, Program Pascasarjana. http://eprints.uny.ac.id/id/eprint/66744

Sumarmo, U., \& Nishitani, I. (2010). High Level Mathematical Thinking: Experiments with High School and Under Graduate Students using Various Approaches and Strategies. In [Natural Sciences Edition] (Vol. 58, pp. 9-22).

Sungur, S., \& Tekkaya, C. (2006). Effects of problem-based learning and traditional instruction on self-regulated learning. Journal of Educational Research, 99(5), 307-320. https://doi. org/10.3200/JOER.99.5.307-320

Tambunan, H. (2019). The Effectiveness of the Problem Solving Strategy and the Scientific Approach to Students' Mathematical Capabilities in High Order Thinking Skills. International Electronic Journal of Mathematics Education, 14(2), 293-302. https://doi.org/10.29333/iejme/5715

Thomas, A., \& Thorne, G. (2009). How to increase higher order thinking. In Metarie, LA: Center for Development and Learning. Center for Development and Learning.(accessed on 6. https:// eric.ed.gov/?id=ED421544

Thompson, T. (2008). Mathematics teachers' interpretation of higherorder thinking in Bloom's taxonomy. International Electronic Journal of Mathematics Education, 3(2), 96-109. http://www. iejme.com/022008/d2.pdf

Thompson, T. (2011). An Analysis of Higher-Order Thinking on Algebra I End-of Course Tests. International Journal for Mathematics Teaching and Learning, c, 1-36.

Turgut, S., \& Turgut, I. G. (2018). The effects of cooperative learning on mathematics achievement in Turkey: A meta-analysis study. In International Journal of Instruction (Vol. 11, Issue 3, pp. 663-680). https://doi.org/10.12973/IJI.2018.11345A

Utomo, S. W., Joyoatmojo, S., Jutmini, S., \& Suryani, N. (2019). Improving Higher Order Thinking Skills Through Problem Based Learning with a Scientific Approach. Dinamika Pendidikan, 14(1), 76-86. https://doi.org/10.15294/dp.v14i1.18776

Vidergor, H. E., \& Krupnik-Gottlieb, M. (2015). High order thinking, problem based and project based learning in blended learning environments. In Applied Practice for Educators of Gifted and Able Learners (pp. 217-232). Brill Sense. https://doi. org/10.1007/978-94-6300-004-8_11

Wardani, I. (2019). Keefektifan Model Guided Inquiry dengan Pendekatan Percobaan Awal terhadap Kemampuan Berpikir Kritis dan Keterampilan Proses Sains Siswa SMA Kelas X Pada Materi Fungi. http://eprints.uny.ac.id/id/eprint/65623

Widiyowati, I. I. (2014). Inovasi Program Pembelajaran Keterampilan Berpikir Kritis Melalui Model Pembelajaran Konstekstual Pada. Seminar Nasional Kimia Kalimantan Timur. https://repository. unej.ac.id/handle/123456789/59395

Yusuf. (2019). Desertasi: Model Pembelajaran Higher Order Thinking Skills pada Siswa Sekolah Menengah Kejuruan. Sekolah Pascasarjanan Universitas Negeri Yogyakarta Indonesia. https://eprints.uny.ac.id/67798/ 